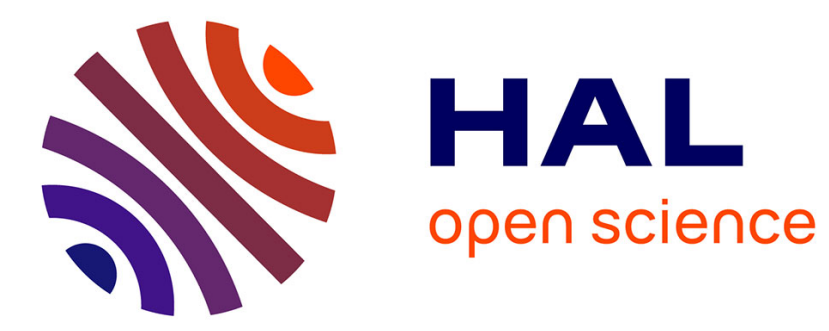

\title{
Sediment erosion in zero-mean-shear turbulence
}

Marie Rastello, Hervé Michallet, Jean-Louis Marié

\section{To cite this version:}

Marie Rastello, Hervé Michallet, Jean-Louis Marié. Sediment erosion in zero-mean-shear turbulence. Physics of Fluids, 2020, 32 (3), pp.036601. 10.1063/1.5141943 . hal-02502263

\section{HAL Id: hal-02502263 https://hal.science/hal-02502263}

Submitted on 9 Mar 2020

HAL is a multi-disciplinary open access archive for the deposit and dissemination of scientific research documents, whether they are published or not. The documents may come from teaching and research institutions in France or abroad, or from public or private research centers.
L'archive ouverte pluridisciplinaire HAL, est destinée au dépôt et à la diffusion de documents scientifiques de niveau recherche, publiés ou non, émanant des établissements d'enseignement et de recherche français ou étrangers, des laboratoires publics ou privés. 


\title{
Sediment erosion in zero-mean-shear turbulence
}

\author{
M. Rastello, ${ }^{1} \mathrm{H}$. Michallet, ${ }^{1}$ and J.L. Marié ${ }^{2}$ \\ ${ }^{1)}$ Univ. Grenoble Alpes, CNRS, Grenoble INP, LEGI, 38000 Grenoble, France. ${ }^{\text {a) }}$ \\ ${ }^{2)}$ Laboratoire de Mécanique des Fluides et d'Acoustique, UMR 5509 \\ Univ. Lyon, CNRS, Ecole Centrale de Lyon, INSA Lyon, Université Claude Bernard Lyon 1, \\ France.
}

(Dated: 27 January 2020)

Turbulence plays an evident role in particle erosion that in many practical situations superimposes with the action of a mean flow. In this paper, the turbulence effect on particle erosion is studied under zero-mean flow conditions, by using the turbulence generated by an oscillating grid. The stirring grid is located more than two mesh size away from the particle layer. The zero-mean flow below the grid has been qualified by revisiting Matsunaga et al. ${ }^{1} k-\varepsilon$ model. The turbulence efficiency on the settling/resuspension of the particles is quantified for various turbulence intensities, varying the size, the nature of the particles, and their buoyancy relative to the fluid. We find that the concentrations $C$ of eroded particles collapse fairly well onto a single trend for $C \leq 5 \times 10^{-2}$, when plotted as a function of the ratio between the flux of turbulent kinetic energy (TKE) at the particle bed location and the particle settling flux. Above, the concentrations saturate thus forming a plateau. Particle erosion mechanisms have been investigated in terms of competing forces within an "impulse approach". Horizontal drag versus friction first leads to a horizontal motion followed by a vertical motion resulting from vertical drag and lift versus buoyancy. Particle erosion occurs when both force balances are in favor of motion for a duration of 0.1 to 0.3 Kolmogorov time scale.

\section{INTRODUCTION}

Sediment erosion and transport are ubiquitous in nature (in rivers, snow avalanches, turbidity currents, snow/sand transport by the wind, beach erosion, sink holes, etc.). Following Shields ${ }^{2}$ work, numerous studies have linked incipient motion and suspension triggering to mean flow properties ${ }^{3}$. Critical thresholds for sediment motion or suspension are indeed generally related to the mean flow bed shear stress reaching specific values dependent on the fluid and particle characteristics. However, these thresholds overlook the fact that natural flows are usually neither uniform, laminar nor really stationary and that the turbulent part of the flow can even be strongly intermittent. Among others Kaftori, Hetsroni, and Banerjee $^{4}$, Niñto and Garcia ${ }^{5}$, Vinkovic et al. ${ }^{6}$ have shown that coherent vortex structures and/or strong turbulent events play a significant role in bed erosion.

In order to study thoroughly the part turbulence plays in the erosion process, we used a well-known experimental device that is the oscillating grid $^{7,8}$. The flow produced by this device has been widely studied ${ }^{1,7,9-12}$. All different studies agree in that far enough from the grid, the mean flow becomes weak enough to be negligible while turbulence appears to be almost isotropic. With a careful design of the grid that helps to inhibit mean secondary flows ${ }^{13}$, the grid oscillating motion generates more than two mesh sizes away from itself turbulence with zero mean flow. Hence, setting up a layer of particles in the tank gives reliable information on erosion processes when turbulence only is involved.

The oscillating-grid generated turbulence (OGT) has regularly been used to investigate a broad range of topics. ${ }^{14-21}$. In the domain of particles flow, Huppert, Turner, and Hallworth ${ }^{14}$, Gratiot, Michallet, and Mory ${ }^{15}$, Matinpour et al. ${ }^{16}$

a)Electronic mail: marie.rastello@legi.cnrs.fr set up the grid to a low position to study highly concentrated suspensions that can exhibit a lutocline formation. Recently, using 3D-PTV and with a high solidity grid set up far from the bottom on which a couple spherical particles were standing, Traugott, Hayse, and Liberzon ${ }^{22}$, Traugott and Liberzon ${ }^{23}$ investigated the forces involved in the lift-off of large particles into turbulent flow.

The originality of the present study is to investigate the particle layer erosion produced by this zero-mean flow OGT for various turbulence intensities, varying the size, the nature of the particles, and their buoyancy relative to the fluid. For that, we use an oscillating grid placed more than two mesh size away from the bottom of the tank on one side and the fluid/air interface on the other, like in Tsai and Lick ${ }^{24}$. The different parameters involved in the problem have been carefully characterized. Turbulence has been quantified by running experiments with fluid only. Erosion runs have been done with a volume of sinking or floating particles set inside the tank prior to the experiment, in order to study the erosion mechanisms on the particle layer by the generated turbulence. Particles and fluid characteristics (size, densities, viscosity) have been varied to widen up the range of investigated non-dimensional numbers (bulk Reynolds number: $R e$, particle Reynolds number: $R e_{p}$, particle to fluid density ratio: $\gamma=\rho_{p} / \rho_{f}$ ).

The paper is built as follows. After presenting the experimental device and the measuring techniques, we draw our attention to the characterization of the flow in the tank. Particle concentration is then investigated. Last part comes with erosion processes and efficiency. 


\section{EXPERIMENTAL SETUP AND PROCEDURES}

\section{A. Experimental setup}

The experimental device is a Perspex tank $(53 \mathrm{~cm} \times 53 \mathrm{~cm} \times$ $90 \mathrm{~cm}$ ). Inside the tank, a stirring grid with square shaped rods (mesh size: $\mathrm{M}=7.5 \mathrm{~cm}$, bar width: $\mathrm{m}=1.5 \mathrm{~cm}$ ) is fixed on a central vertical moving axis (see figure 1). The oscillating mo-

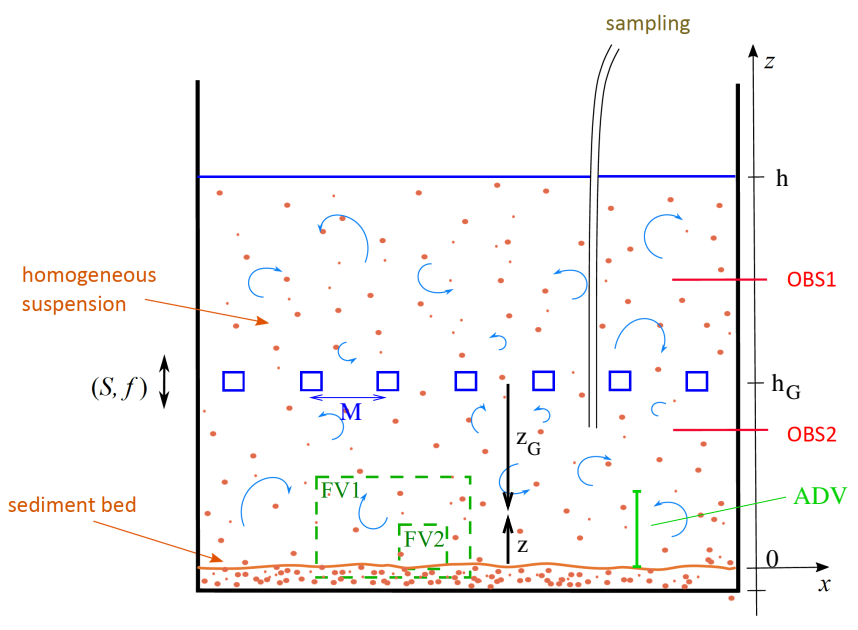

FIG. 1. Sketch of the experimental device with a particle layer at the bottom of the tank. $M$ is the mesh size of the grid, $S$ the Stroke and $f$ the oscillating frequency. $h$ is the total depth of fluid, $h_{G}$ is the mid-position of the grid. $z_{G}$ is the position measured from the grid mid-position while $z$ is measured from the particle layer upper position. The measuring techniques that have been used are indicated: Vertical ADV profiles from $5 \mathrm{~mm}$ above the bottom of the tank up to $10 \mathrm{~cm}$, - PIV on 3 different fields of view: FV1: $14 \mathrm{~cm} \mathrm{x} 10.5 \mathrm{~cm}$, resolution: $0.1 \mathrm{~cm}, \mathrm{FV} 2: 4.7 \mathrm{~cm} \times 3.5 \mathrm{~cm}$, resolution: $0.3 \mathrm{~mm}$ and FV3: $2.4 \mathrm{~cm} \times 1.8 \mathrm{~cm}$, resolution: $0.1 \mathrm{~mm}$. FV3 is not represented in the figure. Concentration is measured with sampling and two optical back-scatter sensors one above the grid (OBS1) and one below (OBS2).

tion (stroke $S$ ) is set with an eccentric coupling, driven by an electric motor through a reducer ${ }^{25}$. The grid mid-position is set prior to the beginning of an experiment. Four categories of experiments (see Table I) have been run investigating no particles, sinking particles and floating particles regimes. Fluid properties have been varied going from pure tap water (experiment $\mathrm{A}$ ) to mixtures of glycerin and water (experiments $\mathrm{B}, \mathrm{C}, \mathrm{D}$ and $\mathrm{E}$ ). In experiments $\mathrm{D}$ and $\mathrm{E}$, the relative density difference is small so that a large amount of particles can be suspended. The distance $h_{G}$ between the grid and the layer of particles varies depending on the forcing.

The volume of fluid was adjusted before running the experiment. A volume of particles was released in the tank for experiments A, C, D and E so that a layer of at least $2 \mathrm{~cm}$ of particles can form, at the bottom of the tank for experiments A, C and D and at the top of the tank for part of experiment E. PolyMethylAcrylate (PMMA) and sawdust particles used in these studies were not mono-disperse particles, the diameter (d) reported is $d_{50} . w_{s}=g^{\prime} d^{2} / 18 v$ is the Stokes settling velocity with $g^{\prime}=g\left(\rho_{p}-\rho_{f}\right) / \rho_{f}$. The mid-position of the grid was chosen as a compromise: not too far from the bed in order to have enough turbulent energy to erode some particles from the layer; not too close to the bed (more than two mesh size away) to avoid mean flow effects that can be easily identified by a wavy surface ${ }^{25}$ of the particle layer. Indeed when mean flow occurs erosion is clearly more pronounced below the rods. One run corresponds to setting up and keeping a constant oscillation frequency $f \leq 6 \mathrm{~Hz}$ of the grid. The minimum duration of a run is 10 minutes (velocity measurements), the maximum one goes up to 60 hours (concentration measurements). Part of the runs with particles were initiated with all the particles in suspension at the beginning of the run ("deposition" run). "Erosion" runs started with the particle layer freshly deposited.

\section{B. Measuring features}

\section{Velocity measurement}

Characterization of the flow was done in multiple ways. It is summarized in Table I . Experiment A refers to experiments run back in 2000 when only homemade acoustic Doppler velocimetry (ADV) sensors $^{26}$ were available in the lab. As a consequence, the measured velocities are less accurate than the ones from experiments B and C. Nevertheless, their qualitative comparison with the model is of some interest (see section III). The probe was held with a rod going through the grid and leaning on the bottom of the tank to reduce vibrations. Velocities were measured from $4 \mathrm{~cm}$ up to $13 \mathrm{~cm}$ above the particle layer in fluid + particles configurations. Velocity measurements lasted 1 minute each in stationary flow conditions. In experiment $\mathrm{B}$, the ADV sensor is a Nortek-ADVVectrino, side-looking, held from above with a rod and immersed in the tank, below the grid. Velocities were measured from $0.5 \mathrm{~cm}$ up to $10 \mathrm{~cm}$ above the bottom of the tank in fluid only configurations, with a minimum of 10 minutes per velocity measurement, in stationary flow conditions. Sampling rate was $100 \mathrm{~Hz}$. In experiment C, Particle Image Velocimetry (PIV) measurements have been performed using a laser $(2 \times$ $200 \mathrm{~mJ}, 532 \mathrm{~nm}$ ) and polyamid round Dantec particles (size: $20 \mu \mathrm{m}$, density: $1.03 \mathrm{~kg} / \mathrm{m}^{3}$ ) together with silver coated Dantec spherical glass particles (size: $10 \mu \mathrm{m}$, density: $1.4 \mathrm{~kg} / \mathrm{m}^{3}$ ) as tracers, depending on the run. The choice of PIV tracers turned to have no influence on the results of the measurements. Images were taken with an Imager Pro X2M /LaVision camera with an acquisition rate of $29 \mathrm{fps}$. Fields of view with different sizes were explored: FV1 $(14 \mathrm{~cm} \times 10.5 \mathrm{~cm}$, resolution: $0.1 \mathrm{~cm}), \mathrm{FV} 2(4.7 \mathrm{~cm} \mathrm{x} 3.5 \mathrm{~cm}$, resolution: $0.3 \mathrm{~mm})$, FV3 $(2.4 \mathrm{~cm} \times 1.8 \mathrm{~cm}$, resolution: $0.1 \mathrm{~mm})$. FV3 is not represented in figure 1 for clarity sake. Part of the runs were done with fluid only, the others with fluid + particles. All the images were including either the bottom of the tank or the upper layers of the particle layer when present (see figure 1). Typical PIV runs lasted 10 minutes in stationary flow conditions. 


\begin{tabular}{|c|c|c|c|c|c|c|c|c|}
\hline Exp & Grid & Fluid & Particles & $R e=f S^{2} / v$ & $R i_{0}$ & $\operatorname{Rou}_{0}$ & $\begin{array}{c}\text { Velocity } \\
\text { measurement }\end{array}$ & $\begin{array}{l}\text { Concentration } \\
\text { measurement }\end{array}$ \\
\hline A & $\begin{array}{c}S=4 \mathrm{~cm} \\
S / M=0.53 \\
h_{G}=17.5 \mathrm{~cm}\end{array}$ & $\begin{array}{c}\text { water } \\
\rho_{f}=1000 \mathrm{~kg} / \mathrm{m}^{3} \\
v=10^{-6} \mathrm{~m}^{2} \mathrm{~s}^{-1}\end{array}$ & $\begin{array}{c}\text { sawdust } \\
\rho_{p}=1060 \mathrm{~kg} / \mathrm{m}^{3} \\
d_{50}=0.5 \mathrm{~mm}\end{array}$ & {$[4.8 ; 9.6] \times 10^{3}$} & $2.1 * 10^{-4}$ & $10^{-2}$ & $\begin{array}{l}\text { side looking } \\
\mathrm{ADV}^{26}\end{array}$ & sampling \\
\hline B & $\begin{array}{c}S=4.3 \mathrm{~cm} \\
S / M=0.57 \\
h_{G}=20.9 \mathrm{~cm}\end{array}$ & $\begin{array}{c}\text { water+glycerin } \\
\rho_{f}=1081 \mathrm{~kg} / \mathrm{m}^{3} \\
v=3 \times 10^{-6} \mathrm{~m}^{2} \mathrm{~s}^{-1}\end{array}$ & No & {$[1.9 ; 3.7] \times 10^{3}$} & No & No & $\begin{array}{c}\text { side looking } \\
\text { ADV (Nortek) }\end{array}$ & No \\
\hline C & $\begin{array}{c}S=4.3 \mathrm{~cm} \\
S / M=0.57 \\
h_{G}=20.9 \mathrm{~cm}\end{array}$ & $\begin{array}{c}\text { water+glycerin } \\
\rho_{f}=1157 \mathrm{~kg} / \mathrm{m}^{3} \\
v=11 \times 10^{-6} \mathrm{~m}^{2} \mathrm{~s}^{-1}\end{array}$ & $\begin{array}{c}\text { plastic (PMMA) } \\
\rho_{p}=1188 \mathrm{~kg} / \mathrm{m}^{3} \\
d_{50}=0.45 \mathrm{~mm}\end{array}$ & {$\left[510 ; 10^{3}\right]$} & $3.6 * 10^{-4}$ & $6 * 10^{-4}$ & PIV & $\begin{array}{c}\text { sampling } \\
+ \\
\text { OBS }\end{array}$ \\
\hline D & $\begin{array}{c}S=4.3 \mathrm{~cm} \\
S / M=0.57 \\
h_{G}=14-17 \mathrm{~cm}\end{array}$ & $\begin{array}{c}\text { water+glycerin } \\
\rho_{f}=1184 \mathrm{~kg} / \mathrm{m}^{3} \\
v=20 \times 10^{-6} \mathrm{~m}^{2} \mathrm{~s}^{-1}\end{array}$ & $\begin{array}{c}\text { plastic (PMMA) } \\
\rho_{p}=1188 \mathrm{~kg} / \mathrm{m}^{3} \\
d_{50}=0.45 \mathrm{~mm}\end{array}$ & {$[50 ; 470]$} & $9.5 * 10^{-3}$ & $2 * 10^{-5}$ & No & $\begin{array}{c}\text { sampling } \\
+ \\
\text { OBS }\end{array}$ \\
\hline E & $\begin{array}{c}S=4.3 \mathrm{~cm} \\
S / M=0.57 \\
h_{G}=13.5-18 \mathrm{~cm}\end{array}$ & $\begin{array}{c}\text { water+glycerin } \\
\rho_{f}=1192 \mathrm{~kg} / \mathrm{m}^{3} \\
v=25 \times 10^{-6} \mathrm{~m}^{2} \mathrm{~s}^{-1}\end{array}$ & $\begin{array}{c}\text { plastic (PMMA) } \\
\rho_{p}=1188 \mathrm{~kg} / \mathrm{m}^{3} \\
d_{50}=0.45 \mathrm{~mm}\end{array}$ & {$[30 ; 370]$} & $9.5 * 10^{-3}$ & $2 * 10^{-5}$ & No & $\begin{array}{c}\text { sampling } \\
+ \\
\text { OBS }\end{array}$ \\
\hline
\end{tabular}

TABLE I. Grid, fluid and particles properties for the different experiments. Grid stirring frequency $f \in[2 \mathrm{~Hz} ; 6 \mathrm{~Hz}]$. Fluid properties are for a temperature of $20^{\circ} \mathrm{C}$. Sawdust particles ${ }^{27}$ are not spherical and have an aspect ratio $\chi=2$. Bulk Rouse number: $\operatorname{Rou}_{0}=w_{s} / \sqrt{k}_{0}$ with $w_{s}=g^{\prime} d^{2} / 18 v$ : the Stokes settling velocity, $k_{0}$ : typical turbulent kinetic energy at the virtual origin of the grid. Bulk Richardson number ${ }^{28}$ : $R i_{0}=g^{\prime} C_{0} \sqrt{k_{0}} / \varepsilon_{0}$ with $g^{\prime}=g\left|\rho_{p}-\rho_{f}\right| / \rho_{f}, C_{0}$ : typical particle volumetric concentration and $\varepsilon_{0}$ : typical dissipation at the virtual origin of the grid. The two right-hand side columns list the velocity and concentration measuring techniques, respectively.

\section{Concentration measurement}

The techniques used to measure the volumetric concentration in particle are summarized in Table I. In experiment A concentration was monitored through sampling: 1 to $2 \mathrm{~L}$ of a fluid + particles mixture was pumped out of the tank using a peristaltic pump, 20 minutes after the experiment had begun out of a freshly deposited layer of particles. Concentration had been verified to be homogeneous in space under the grid and stationary in time at sampling time. Filtration of the particles followed by a careful drying using a drying chamber was performed. Weight measurement was then performed with a scale. Experiments C, D and E were performed in long runs (up to 60 hours) so to reach steady states ${ }^{29}$. Two optical back-scatter sensors (OBS-3+/Campbell Scientific) one located just below the lowest position of the grid and the other $10 \mathrm{~cm}$ above the first one were used to monitor the time evolution of the concentration in the tank (see figure 1). The acquisition rate was $4 \mathrm{~Hz}$. Sampling at the height of the lower OBS was performed as well so as to provide a calibration curve for the OBS. Sampling was handled in the same way as for sawdust particles.

\section{CHARACTERIZATION OF THE FLOW}

In all our experiments the product of the bulk Rouse number with the bulk Richardson number $R i_{0} R o u_{0} \ll 1$. As a consequence, according to Michallet and Mory ${ }^{28}$, turbulence decay is not affected by particle concentration far from boundaries. Flow characteristics are analysed in light of the results of Mat- sunaga et al. ${ }^{1}$ who investigated experimentally the turbulence field generated by an oscillating grid and proposed a $k-\varepsilon$ model consistent with the existing correlations of Hopfinger and Toly ${ }^{7}$, Ura, Komatsu, and Matsunaga ${ }^{30}$, Silva and Fernando ${ }^{31}$. Their situation differs from ours since the turbulence generated does not interact with a solid floor or particle layer like here, but develops towards the water surface. The comparison is therefore interesting. However applying this model to the range of viscosity $\left(v \in\left[10^{-6} ; 10^{-5}\right] \mathrm{m}^{2} . \mathrm{s}^{-1}\right)$ and bulk Re number covered in our study (Table I) gives rise to some inconsistencies. Indeed, for higher viscosity fluids (low bulk Re), TKE is decreasing less rapidly from the grid than for lower viscosity fluids, making the corresponding $k$ versus $z_{G}$ curves (higher viscosity and lower viscosity ones) crossing for $z_{G} / M \approx 1-1.5$. This would imply a larger TKE far from the grid for more viscous fluids than for less viscous ones. This problem results from the data chosen in the figure 10 of Matsunaga et al. ${ }^{1}$ to derive the $R e$ dependency of the turbulent energy $k_{0}$ and the dissipation rate $\varepsilon_{0}$ that are taken as virtual boundary conditions in their model. The data can be classified into three categories. The values measured by the authors for $R e \geq 300$ where the flow generated by the grid can be considered as fully turbulent. The values measured for $\operatorname{Re}<300$ where it is probably weakly turbulent and the measurements of dissipation can be questionable. At last, the data stated as borrowed to Thompson and Turner ${ }^{32}$ and Ura, Komatsu, and Matsunaga $^{30}$. We chose to discard these two references, the first because no dissipation measurements were reported in the paper, the second as appearing as less complete and reliable. Retaining only the data of Matsunaga et al. ${ }^{1}$ yields figure 2 . The new correlations proposed for $k_{0}$ and $\varepsilon_{0}$ in attributing a 


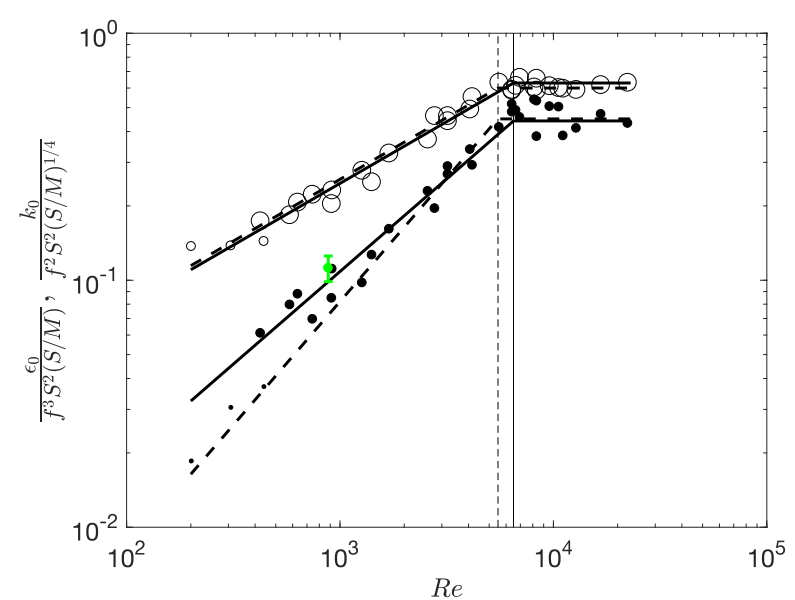

FIG. 2. Digitized Matsunaga's data: large symbols: $R e \geq 300$, small symbols: $R e<300$. o : values linked with $k_{0}$, $\bullet$ : values linked with $\varepsilon_{0}$. $\bullet$ : dissipation value and error bar measured at $\mathrm{f}=5 \mathrm{~Hz}$ from experiment C FV1-PIV fields. Black oblique/horizontal lines: equation 1, 3 and 2 and 4, dashed oblique/horizontal lines: Matsunaga original fits. Vertical lines materialize the $R e$ transition value (see equation 1, 3 and 2 and 4), plain line: present model, dashed line: original Matsunaga's model.

higher degree of confidence to the data $R e \geq 300$ where the flow can be considered as "fully turbulent" come as follows: For $\operatorname{Re}<6.5 \times 10^{3}$ :

$$
\begin{gathered}
k_{0}=7.8 \times 10^{-3} f^{2} S^{2}(S / M)^{1 / 4} R e^{1 / 2} \\
\varepsilon_{0}=6.1 \times 10^{-4} f^{3} S^{3} / M \times R e^{3 / 4}
\end{gathered}
$$

For $R e \geq 6.5 \times 10^{3}$ :

$$
\begin{gathered}
k_{0}=0.63 f^{2} S^{2}(S / M)^{1 / 4} \\
\varepsilon_{0}=0.44 f^{3} S^{3} / M
\end{gathered}
$$

with

$$
z_{0}=\frac{k_{0}^{3 / 2}}{\varepsilon_{0}}
$$

Keeping the other equations of the model unchanged:

$$
\begin{aligned}
& k=k_{0} \times\left(\frac{z_{G}}{1.82 z_{0}}+1\right)^{-5} \\
& \varepsilon=\varepsilon_{0} \times\left(\frac{z_{G}}{1.82 z_{0}}+1\right)^{-8.5}
\end{aligned}
$$

This new formulation ensures that the "crossing" effect is not occurring anymore and that TKE decreases more rapidly for higher viscosity fluids than for lower viscosity fluids, as expected. The model of Matsunaga et al. ${ }^{1}$ thus corrected and
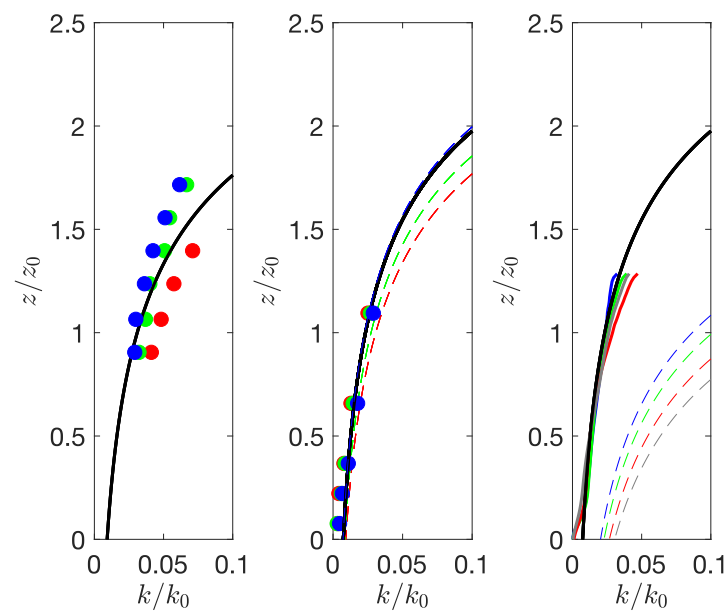

FIG. 3. Variation of the turbulent kinetic energy ratio with dimensionless distance from the boundary $\left(z / z_{0}\right)$. Left: Experiment A, middle: experiment $B$, right: experiment $C$. Blue: $f=6 \mathrm{~Hz}$, green: $f$ $=5 \mathrm{~Hz}$, red: $\mathrm{f}=4 \mathrm{~Hz}$, grey: $\mathrm{f}=3 \mathrm{~Hz}$. Black plain line: modified model with equation 1, 3 and 2 and 4 , dashed lines: Matusnaga's model.

validated for low-grid-position configurations has been tested on our measurements in a high-grid-position configuration. Figure 3 shows the modified version of the $k-\varepsilon$ model compared with experiments $\mathrm{A}, \mathrm{B}$ and $\mathrm{C}$ performed with various viscosity and bulk Re numbers (cf Table I). The agreement between experimental data far enough from the boundary and the new version of the model can be observed for the three bulk-Re ranges covered. As viscosity increases and bulk Re number decreases $\left(R e \leq 6.5 \times 10^{3}\right)$ the discrepancy with the initial model ${ }^{1}$ increases and becomes substantial. The FV1PIV data of experiment $\mathrm{C}$ has been used to compute spatial turbulent kinetic energy spectra in both the horizontal and vertical direction in the bulk part of the flow ${ }^{33}$. These PIV fields cover about 2-3 integral scales with a resolution of $1.4 \mathrm{~mm}$ of the order of the Kolmogorov scale $(2-3 \mathrm{~mm})$. These scales were estimated from Gratiot, Michallet, and Mory ${ }^{15}$ and Matsunaga et al. ${ }^{1}$, respectively. Hence, the largest wave numbers (dissipative scales) as the smallest wave numbers (integral scales) are not accurately resolved in the spectra. Despite this loss of resolution, as can be seen in figure 4 , a small $-5 / 3$ inertial region is visible even for the intermediate $R e(1000)$ corresponding to flows with a higher viscosity. We estimated the dissipation rate from the second-order longitudinal structure function ${ }^{33,34}$ spatially averaged at different altitudes of the domain of homogeneity. Results are plotted in figure 5. Only data where an inertial subrange on the structure function could be identified without any ambiguity has been reported. It corresponds to regions far from the bed where the flow can be considered as quasi homogeneous isotropic. Resulting $\varepsilon(z)$ follow quantitatively the modified version of Matsunaga et al. ${ }^{1}$ model rather well, confirming the need of modification especially for more viscous fluids. The values of $\varepsilon_{0}$ corresponding to the $(z, \varepsilon(z))$ measured are obtained using relations (1), (2), (5), (7). Average and standard deviation is reported in figure 2. Figure 6 confirms that far from the grid (more than 


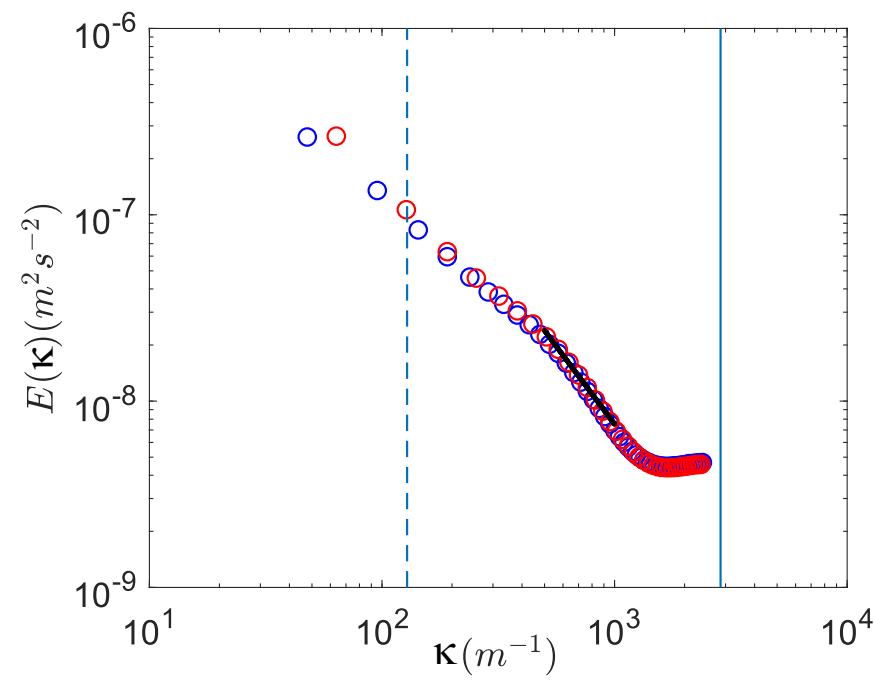

FIG. 4. Spatial turbulent kinetic energy spectrum (experiment C FV1-PIV fields; $f=5 \mathrm{~Hz})$. blue $\circ$ : horizontal direction $\left(E_{u u}\right)$, red $\circ$ : vertical $\left(E_{w w}\right)$ direction. Blue dotted line: estimate of the integral length scale $\kappa_{i n t}$; blue solid line: estimate of the Kolmogorov one: $\kappa_{\eta}$; black solid line: inertial range trend $\kappa^{-5 / 3}$.

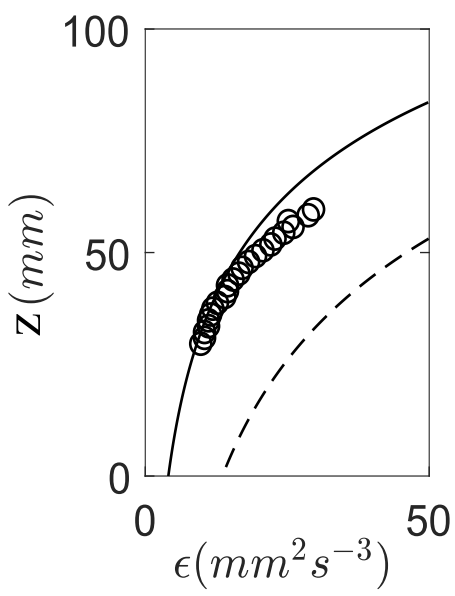

FIG. 5. Dissipation as a function of the distance from the boundary (experiment C FV1-PIV fields; $f=5 \mathrm{~Hz}$ ). ०: measurements, plain line: new version of the model, dashed line: original version of the model.

two mesh size away) and far enough from the boundary (more than $0.2 z_{0}$ ) the flow is fairly isotropic. As expected, close to the boundary, the structures get deformed and flatten inducing an increase in $\sigma_{u}, \sigma_{v}$ relatively to $\sigma_{w}$. The behavior very close to the boundary when velocities go back to zero was not reachable with the precision of the study.

\section{PARTICLE CONCENTRATION}

Concentration in particles during transient states has been addressed in Rastello, Michallet, and Marié ${ }^{29}$. In the region between the grid and the free surface Michallet and Mory 28
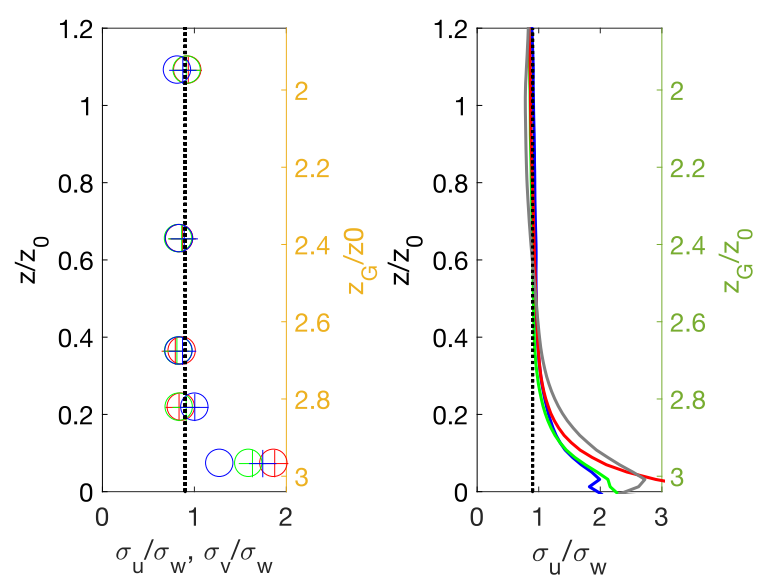

FIG. 6. Variation of the ratio between the turbulent horizontal velocities and the vertical one $\left(\sigma_{u} / \sigma_{w}, \sigma_{v} / \sigma_{w}\right)$ with dimensionless distance from the grid $\left(z_{G} / M\right.$ and from the boundary $\left.z / M\right)$. Left: experiment B: O: $\sigma_{u} / \sigma_{w},+: \sigma_{v} / \sigma_{w}$, right: experiment $\mathrm{C}, \sigma_{u} / \sigma_{w}$. Blue: $f=6$ $\mathrm{Hz}$, green: $f=5 \mathrm{~Hz}$, red: $f=4 \mathrm{~Hz}$, grey: $f=3 \mathrm{~Hz}$.

have shown that when $\mathrm{Rou}_{0}<0.01$, particle concentration is homogeneous in the whole region aside from very close to the grid and beyond a possible lutocline. This homogeneous concentration Rou domain should reasonably be the same for our flow below the grid. Experiments A, C, D and E (see Table I) exhibit favorable values of Rou. Sampling in multiple different points together with the two OBS measurements have confirmed that concentration in particles is homogeneous in the whole tank at steady state for all three types of runs. As a consequence, concentration values come from a single location measurement (sampling or OBS2). No clear dependency on the initial state (particles deposited or in suspension before the run) has been noticed. As a consequence, runs were performed indifferently with both initial state being a brand new deposited particle layer ("erosion run") or a state with all the particles in suspension at start ("deposition run").

Without any mean flow, the energy available to lift up a particle from the bed is the kinetic energy of the turbulent structures approaching the surface of the bed. This kinetic energy could hardly be estimated from our velocity measurements because in some case these data were missing like in experiment $\mathrm{D}$ or $\mathrm{E}$ or were not accurate enough like in experiment A (see Table I). It was therefore inferred from the modified model of Matsunaga et al. ${ }^{1}$. The use of the original model would greatly over predict the turbulent energy potentially available for lift up. This energy was estimated at $z=z_{l}=20 \mathrm{~mm}$. This choice seems reasonable because it is the thickness of the boundary layer over the bed where turbulence gradually loss its isotropy (see figures 6 and 3-right). The turbulent energy at this altitude can thus be viewed as the energy of the turbulent structures entering this boundary layer and likely to contribute to the particle lift up. The resulting turbulent flux $T$ entering the boundary layer and that will promote particle lift-off is compared to the settling flux $g^{\prime} w_{s}$ that deposits particles in figure 7. $T$ can be estimated using a k- $\varepsilon$ 
model $^{1,28}$ as

$$
T=-\frac{1}{z_{l}}\left(v+0.09 \frac{k^{2}}{\varepsilon}\right) \frac{d k}{d z}
$$

Ratio $T / g^{\prime} w_{s}$ appears to rule concentration as data from multiple different runs collapse fairly well onto a single trend

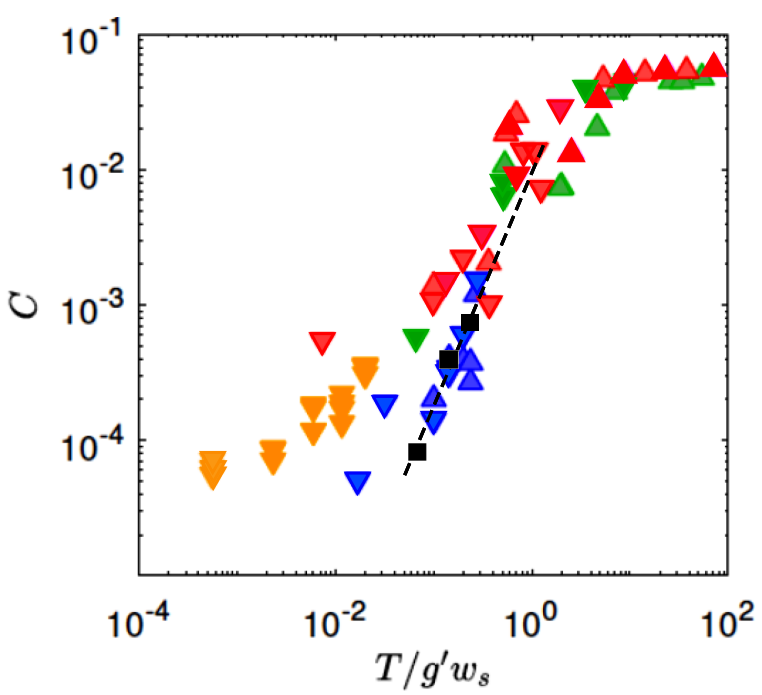

FIG. 7. Concentration as a function of the ratio between turbulent and settling fluxes. $\triangle:$ deposition runs, $\nabla$ : erosion runs. Yellow: experiment $\mathrm{A}$, blue: experiment $\mathrm{C}$, green: experiment $\mathrm{D}$, red: experiment $\mathrm{E}$. Black squares, concentration values given by the erosion "impulse approach" (see section V); dashed line: increasing trend deduced from these values.

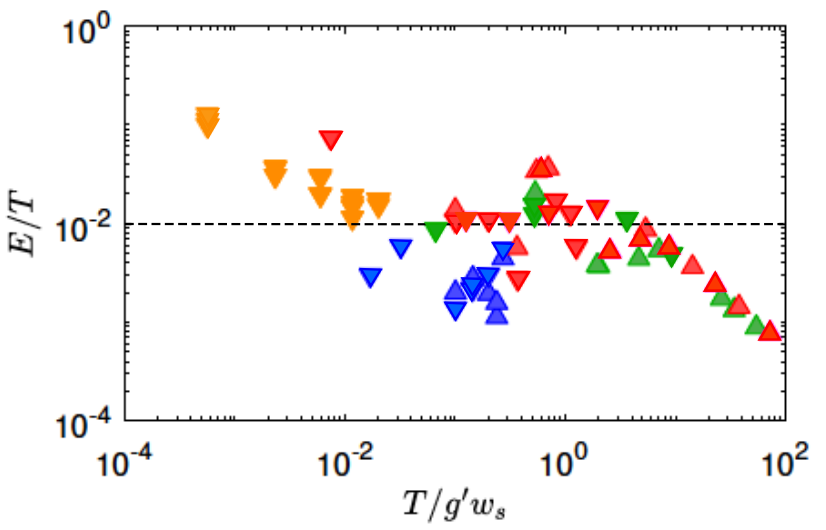

FIG. 8. Erosion flux over turbulent flux as a function of the ratio between turbulent and settling fluxes. $\triangle:$ deposition runs, $\nabla$ : erosion runs. Yellow: experiment $\mathrm{A}$, blue: experiment $\mathrm{C}$, green: experiment $\mathrm{D}$, red: experiment $\mathrm{E}$.

over more than two decades of concentration $\left(C \approx\left[10^{-4} ; 5 \times\right.\right.$ $\left.\left.10^{-2}\right]\right)$ and flux ratios $\left(T / g^{\prime} w_{S} \approx\left[5 \times 10^{-3} ; 5\right]\right)$. Concentration values below $10^{-4}$ are to be taken with more care given the very low amount of particles in suspension. The larger concentration values $\left(C>5 \times 10^{-2}\right)$ appear to saturate in a plateau. Given the high value of concentration it sounds reasonable to expect collective effects for these runs that could affect both erosion process, settling velocity and turbulence as reported in Gratiot, Michallet, and Mory ${ }^{15}$ with cohesive sediments. The change of trend could reasonably be linked to it. The corresponding ratio between erosion and turbulent fluxes is plotted in figure 8 with the erosion flux being

$$
E=g^{\prime} C w_{s}
$$

The 3 regions identified in figure 7 are also visible. In the central region where all data collapse following the increasing trend, erosion flux is proportional to turbulent flux $\left(10^{-2}\right.$ plateau in figure 8). The decrease observed above $T / g^{\prime} w_{S}>5$ is linked to the potential collective effects previously mentioned. The overall scattering of the data in the central region of figures 7 and 8 can be attributed to various factors. The determination of $g^{\prime}$ is less accurate for experiments D and E, where $\rho_{p}$ and $\rho_{f}$ have close values. Of note, $\rho_{f}$ depends on the temperature, that was monitored in order to minimize any bias. The distance between the position of the grid and the particle layer can vary from an experiment to another, so that the existence of a possible weak residual mean flow may have an impact. Also the particles shape and the size dispersion around $d_{50}$ may contribute to the scattering.

\section{EROSION PROCESS AND EFFICIENCY}

Erosion process when not coming from mostly mean flow is still an open topic. Traugott, Hayse, and Liberzon ${ }^{22}$, Traugott and Liberzon ${ }^{23}$ performed experiments in a turbulence generated by a stirring grid, with a few silica gel particles on a smooth boundary (density comparable to the fluid, diameter comparable with the Kolmogorov length scale, $R e_{p}=\frac{w_{s} d}{v}=$ 6 ). The pattern of the grid was modified to increase its solidity and its mid-position adjusted to less than two mesh size from the bottom of the tank. As a consequence, their incoming flow was a mix of turbulence generated by the grid movement and remaining mean flow. From their study it comes up that first the particles were set in a horizontal motion. While moving horizontally some particles were then lifted up. By carefully monitoring the flow around the particles the authors have shown that to experience a so-called lift-off, the particles had to experience a significant increase of the relevant forces during a finite amount of time. In their configuration the order of magnitude of the duration was 0.5 to 0.7 times Kolmogorov time. They linked their results to Celik et al. ${ }^{35}$ who suggested that the time interval of application of the force acting on the particle is important when predicting whether or not a particle will be entrained by a turbulent flow event ("impulse approach").

Munro, Bethke, and Dalziel ${ }^{36}$, Bethke and Dalziel ${ }^{37}$ have conducted experiments with an impacting vortex on a particle layer in a surrounding still fluid. The particles used had a density going from 1.2 to 7 times the fluid density and sizes ranging from $90 \mu m$ to $1100 \mu m\left(\operatorname{Re}_{p} \in[1 ; 300]\right)$. From their studies it appears that as the vortex approaches the particle layer, the 
particles are set in a sliding motion towards the edges of the vortex where they are then lifted up in the region in between the primary vorticity zone and the second counter-rotating one created at the edges of the traveling vortex by the presence of the solid interface/particle layer. The behavior can be qualitatively observed on the supplemental video resources on the journal 2012 paper website.

The turbulence that our device is generating can be seen as a superposition of turbulent eddies (vortices) of different sizes/strengths traveling towards the bottom of the tank. Video and PIV analysis of the erosion process in the present setup indicate that first particles are set into a sliding/rolling motion and then after a certain duration of this horizontal motion are lifted up in suspension. This is illustrated by the FV3-PIV time sequences given in figure $9 \mathrm{a}, \mathrm{b}$. Sequence (a) shows a particle set into a horizontal motion by the drag generated by the horizontal flow, while sequence (b) corresponds to another particle that starts a vertical motion (lift up). This particle initially at rest (Figure $9 \mathrm{~b}$-left) starts a lift up within the first second after being set into motion by the previous mechanism.
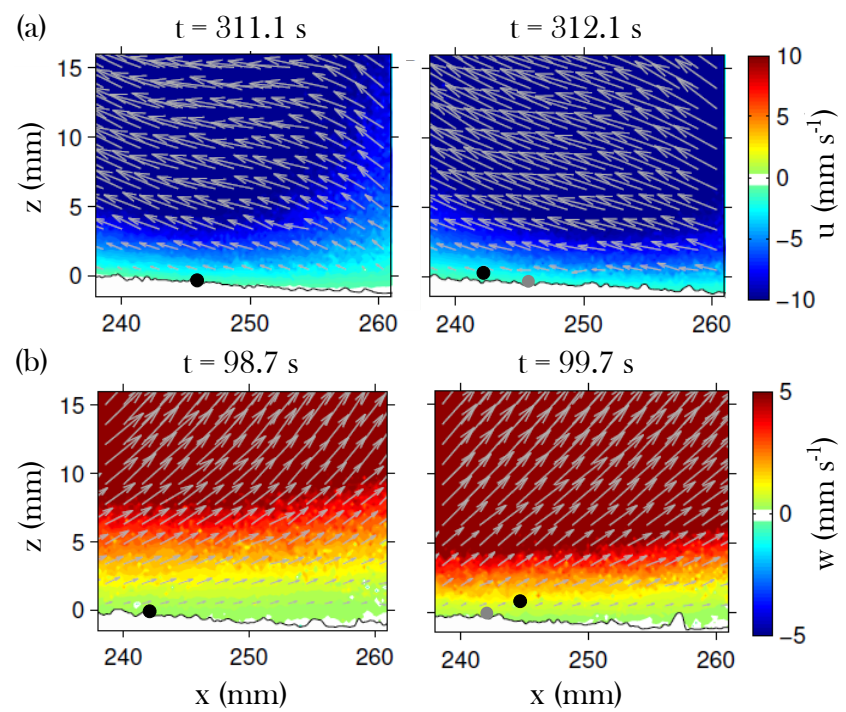

FIG. 9. Time series exhibiting the flow induced by the turbulent eddies impacting on the bed and its typical effect on the particles motion. $f=5 \mathrm{~Hz}$. Black circles materializes the particle positions. Grey circles (right figures) are the initial positions before displacement. (a) a particle set into a sliding/rolling horizontal motion by the horizontal drag. Horizontal velocity at the top of the boundary layer $u_{\delta}>2 \mathrm{~mm} / \mathrm{s}$. (b) Another particle starting its lift up after being set into horizontal motion. Horizontal velocity at the top of the boundary layer $u_{\delta}>2 \mathrm{~mm} / \mathrm{s}$, vertical velocity at the top of the boundary layer $w_{\delta}>1 \mathrm{~mm} / \mathrm{s}$.

When set up in suspension the particles are, at first, having an oblique trajectory that evokes the trajectories of the particles trapped between the two vortical regions mentioned in Munro, Bethke, and Dalziel ${ }^{36}$, Bethke and Dalziel ${ }^{37}$ (see for instance figure 10). From these observations a criterion for particles being set up in suspension has been investigated through a coupled mechanism: the particles are first set into
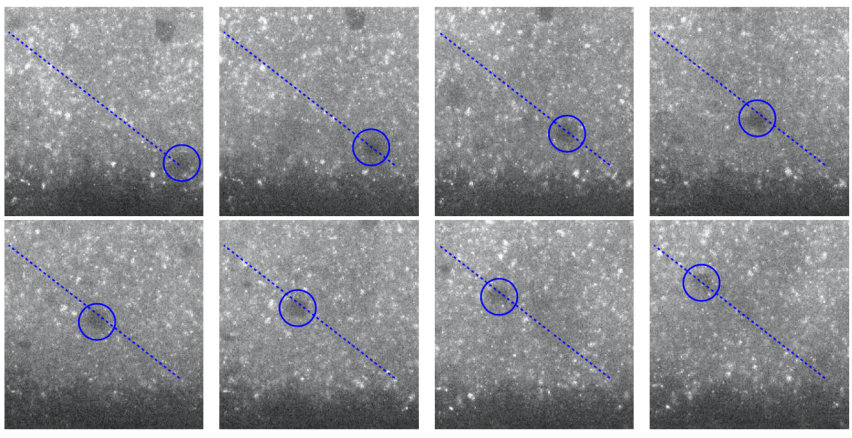

FIG. 10. Erosion of a particle from the particle layer and early instants after lift up exhibiting the typical oblique trajectory observed. The blue line showing the direction is at the same location on each image. Time laps between 2 successive images is $0.14 \mathrm{~s}$. Particle velocity: $3.5 \mathrm{~mm} \cdot \mathrm{s}^{-1}$. Image size is $4 \mathrm{~mm} \times 4.5 \mathrm{~mm}$.

a sliding/rolling motion for a certain duration $\mathscr{T}$ (value to be determined), then these particles are lifted up. For both the onset of the horizontal motion (h) and the suspension one (s) a critical Shields number is defined as

$$
\theta_{c / h, s}=\frac{u_{\delta / h, s}^{2}}{(\gamma-1) g d}
$$

with $u_{\delta / h, s}$ the critical value of the horizontal velocity at the top of the boundary layer for either onset of horizontal motion or vertical one. Our particles are embedded in the viscous sublayer (see section $\mathrm{VC}$ ). $u_{\delta}$ is chosen instead of $u_{d}$ (velocity at the position of the particle, $z=d$ ) since the corresponding velocity measurements are more accurate.

\section{A. Onset of horizontal motion}

Among others Loiseleux et al. ${ }^{38}$, Ouriemi et al. ${ }^{39}$ worked on the onset erosion criterion for particle erosion by a laminar flow. From their work it results that on a horizontal boundary to have particles set in horizontal motion the horizontal drag has to be greater than the friction force which results in

$$
u_{d} \geq \frac{\mu_{e}}{\alpha^{\prime}} w_{s}
$$

with $\mu_{e}$, the apparent friction coefficient that takes into account solid friction together with roughness and traps of the surface. $\alpha^{\prime}$ is a constant that takes into account that the particle is not alone on a solid boundary but part of a layer of particles. For particles fully immersed in the viscous sublayer of a turbulent flow $(d \leq \delta)$ a laminar flow type analysis for the onset in horizontal motion is likely to be used as well. The viscous boundary layer thickness $\delta$ expresses as $v / u_{\delta}$, while the horizontal velocity inside the layer is linear: $u_{d}=u_{\delta} d / \delta$. The criterion for the onset of horizontal motion, as given by (10) then writes

$$
u_{\delta}=\sqrt{\frac{\mu_{e} v}{\alpha^{\prime} d} w_{s}}
$$


which leads to a critical Shields number

$$
\theta_{c h}=\frac{\mu_{e}}{18 \alpha^{\prime}}
$$

that is independent of $R e_{p}$ as long as the particle remains embedded in the viscous sublayer.

\section{B. Particle lift up}

Munro, Bethke, and Dalziel ${ }^{36}$, Bethke and Dalziel ${ }^{37}$ studied the lift up criteria that can be exhibited for a particle originally staying on a horizontal boundary. They first considered the case in which the particle is bigger than the viscous sublayer $(d \geq \delta)$ and experiences the flow at the top of the boundary layer. From the balance between the lift force and the buoyancy they ended in a constant critical Shields number for lift up. On the other hand, when the particle is smaller than the viscous sublayer size $(d \leq \delta)$ the equilibrium between lift force and buoyancy force gave them a critical Shields number that decreases in $R e_{p}^{-1 / 2}$.

Using the same kind of approach we investigated the forces experienced by our particles embedded in the viscous sublayer (see section VC). To the lift and buoyancy forces used by Munro, Bethke, and Dalziel ${ }^{36}$ and Bethke and Dalziel ${ }^{37}$ we added the possible impact of the vertical drag force in the balance. The forces in presence are then - Lift force ${ }^{36}$

$$
F_{L} \approx \pi \rho_{f} u_{d}^{2} d^{2}
$$

- Vertical drag force:

$$
F_{D}=3 \pi \rho_{f} v d w_{d} \alpha
$$

with $w_{d}$ the vertical fluid velocity experienced by the particle and $\alpha$ a parameter taking into account that the particle is sitting on a solid boundary and not in the middle of the fluid far from boundaries ${ }^{40}$.

- Buoyancy force:

$$
F_{B}=\frac{\pi(\gamma-1) \rho_{f} g d^{3}}{6}
$$

As expected, comparing lift and buoyancy ends in the same results as reported in the previous studies: $\theta_{c s} \sim \operatorname{Re}_{p}^{-1 / 2}$. Since $F_{L} \sim u_{d}^{2} d^{2}$ and $F_{D} \sim w_{d} d$, when the particle diameter decreases the lift force decreases more rapidly than the drag does to a point where lift becomes negligible in front of the drag for small enough particles. This leads to different balances depending if lift or vertical drag has to be considered versus buoyancy. The ratio between lift and drag comes as

$$
\frac{F_{L}}{F_{D}} \sim A \frac{d}{\delta}
$$

where

$$
A=\frac{\beta}{3 \alpha}
$$

is a key parameter to quantify the relative importance of the two forces, $\beta=u_{\delta} / w_{\delta}$. The expression of A can be readily obtained from the linearity of the velocity profile in the viscous layer $\left(u_{d}=u_{\delta} d / \delta\right)$ and the continuity equation that yields

$$
w_{d}=\frac{d^{2}}{\delta^{2}} w_{\delta}
$$

Depending on the kind of flow and thus on the ratio between the two velocities $\left(u_{\delta}\right.$ and $\left.w_{\delta}\right)$, the major forces in balance change. The various flow situations that may be encountered are summarized in Table II. In the case where drag balances

\begin{tabular}{|l|l|l|}
\hline A, Size & Forces & Suspension criteria \\
\hline$A \leq 1$ & $\begin{array}{l}\text { Drag } \\
+\end{array}$ & $\begin{array}{l}\theta_{c s}=\frac{1}{18}\left(\frac{\beta^{2}}{\alpha^{2} R e_{p}}\right)^{1 / 3} \\
\text { and } \\
\end{array}$ \\
\hline $\begin{array}{l}\text { Buoyancy } \\
w_{d}=w_{s}\end{array}$ & Drag & $\theta_{c s}=\frac{1}{18}\left(\frac{\beta^{2}}{\alpha^{2} R e_{p}}\right)^{1 / 3}$ \\
and & + & and \\
$A \frac{d}{\delta} \leq 1$ & Buoyancy & $\alpha w_{d}=w_{s}$ \\
\hline$A \geq 1$ & Lift & $\theta_{c s} \approx R e_{p}^{-1 / 2}$ \\
and & + & \\
$A \frac{d}{\delta} \geq 1$ & Buoyancy & \\
\hline
\end{tabular}

TABLE II. Summary of the forces involved and suspension criteria depending on the size of the particle and the characteristics of the boundary layer for a particle immersed in the boundary layer.

buoyancy (cf Table II), the critical Shields number varies as $\operatorname{Re}_{p}^{-1 / 3}$ against $\operatorname{Re}_{p}^{-1 / 2}$ when lift is prevailing. Munro, Bethke, and Dalziel ${ }^{36}$, Bethke and Dalziel ${ }^{37}$ found in their figure $6 \mathrm{a}$ (2009 paper) and 7b (2012 paper) that a $R_{p}^{-1 / 2}$ dependence did not seem to fit well their data and that a fit with a -0.37 exponent was better. This value is close to $-1 / 3$. This suggests that for the type of particles and flow they investigated, the particles behavior is probably more reasonably governed by drag and buoyancy. This would explain the discrepancy they had when using lift and buoyancy to interpret their measurements.

\section{Horizontal motion and particle lift up criteria in the present experiment}

We used the criteria proposed previously to estimate the concentration in eroded particles in the present setup. Experiment $\mathrm{C}$ being the more complete (PIV + concentration measurement) has been used. For the particles used (PMMA): $d=0.45 \mathrm{~mm}$. The viscous sublayer thickness can be estimated from the PIV velocity profiles as: $\delta \approx \frac{v}{u_{\delta}} \approx 3 \mathrm{~mm}$. As a consequence, the particles are completely immersed in the viscous sublayer. Thus, (11) is valid to estimate the onset of our horizontal particle motion. Following Loiseleux et al. ${ }^{38}$ 
$\frac{\mu_{e}}{\alpha^{\prime}} \approx 1$ in (10) which substituted in (11), leads for this onset

$$
u_{d}=w_{s} \Longleftrightarrow u_{\delta}=\sqrt{\frac{v w_{s}}{d}} \approx 2 \mathrm{~mm} / \mathrm{s}
$$

and

$$
\theta_{c h} \approx \frac{1}{18}
$$

In figure 9-a, horizontal motion initiation happens with $u_{\delta} \geq$ $2 \mathrm{~mm} / \mathrm{s}$.

To know which force balances buoyancy in the initiation of vertical motion, parameter A needs to be estimated. From the PIV profiles obtained in our study it is possible to estimate that $\beta=u_{\delta} / w_{\delta} \approx 2-4$ (see figure 6-right). Parameter $\alpha$ is more delicate to evaluate. According to Guyon et al. ${ }^{40}$, drag perpendicular to a solid boundary layer is doubled close to the boundary layer, suggesting that $\alpha \approx 2$. This yields a value of $A \leq 1$ representative of an equilibrium between drag and buoyancy (cf Table II). This leads to

$$
w_{d}=\frac{w_{s}}{\alpha} \approx \frac{w_{s}}{2} \Longleftrightarrow w_{\delta}=\left(\frac{v^{2} w_{s}}{\alpha \beta^{2} d^{2}}\right)^{\frac{1}{3}} \approx 1 \mathrm{~mm} / \mathrm{s}
$$

for the fluid vertical velocity to set a particle in suspension and

$$
\theta_{c s} \approx \frac{1}{18 \operatorname{Re}_{p}^{1 / 3}}
$$

At ejection instants in Figure $9 \mathrm{~b}, w_{\delta}>1 \mathrm{~mm} / \mathrm{s}$. In figure 10, the particle is ejected with an overall velocity of the order of $3.5 \mathrm{~mm} / \mathrm{s}$ and a vertical velocity of $2.4 \mathrm{~mm} / \mathrm{s}$ qualitatively coherent with the lift up threshold of $w_{\delta}=u_{\delta} / \beta \approx 1 \mathrm{~mm} / \mathrm{s}$.

Referring to Traugott, Hayse, and Liberzon ${ }^{22}$, Traugott and Liberzon $^{23}$, Celik et al. ${ }^{35}$, Munro, Bethke, and Dalziel ${ }^{36}$, Bethke and Dalziel ${ }^{37}$, to be lifted up by the flow, the particle needs to be exposed during a specific duration $\mathscr{T}$ to a flow favoring both a horizontal movement and a lift up ("impulse approach"). In other words to have a particle set up in the vertical erosion process, both the horizontal movement and lift up criteria (see (18) and (20)) need to be verified by the flow close by the particle for a duration of at least $\mathscr{T}$.

To test the validity of the "impulse criterion" hypothesis for the present study we counted the number of particles that were eroded during a recording-time $\Delta \mathscr{T}=10 \mathrm{mn}$ on the FV3-PIV sequences of experiment $\mathrm{C}$. For a grid oscillating frequency of $5 \mathrm{~Hz}$, the tracking of the particles ejected from the bed surface $s=24 \mathrm{~mm} \times 1 \mathrm{~mm}$ region located at the intersection with the laser sheet (thickness $1 \mathrm{~mm}$ ) ends in on average 30 eroded particles. This number is consistent with the value deduced from the concentration. Indeed, in stationary conditions, the settling $(S)$ and erosion $(E)$ fluxes balance (Rastello, Michallet, and Marié $\left.{ }^{29}\right)$ and are expressed as

$$
S=E=g^{\prime} w_{s} C
$$

It follows that the number $n_{p}$ of particles deposited/eroded from the same bed surface $s$ within the laser sheet during the same $\Delta \mathscr{T}=10 \mathrm{mn}$ recording-time is

$$
n_{p} \approx \frac{w_{s} C s \Delta \mathscr{T}}{V_{p}}
$$

$V_{p}$ being the average volume of the particles. In experiment $\mathrm{C}$, concentration in particles for $f=5 \mathrm{~Hz}$ is $C_{5 \mathrm{~Hz}} \approx 4 \times 10^{-4}$ (cf figure 7)) while $w_{s}$ and $V_{p}$ are given by Table I. It yields a number of deposited/eroded particles $n_{p \_5 \mathrm{~Hz}}=33$ of the same order of magnitude than the number previously obtained. We deduce that $n_{p_{-} 5 \mathrm{~Hz}} \approx 30$ is a reasonable estimation of the deposited/eroded particles for this situation. Knowing this number, the number of favorable events at $5 \mathrm{~Hz}\left(n_{f e} 5 \mathrm{~Hz}\right)$ where both criteria (18) and (20) are fulfilled during a specified duration $\mathscr{T}$ have been determined from the same FV3-PIV sequences. As expected, the number of favorable events for erosion detected strongly varies with the duration $\mathscr{T}$ considered. Duration $\mathscr{T}$ for which the number of favorable events $n_{f e_{-} 5 \mathrm{~Hz}}$ is of the same order of magnitude as the number of particle eroded $n_{p_{-} 5 \mathrm{~Hz}}$ in $10 \mathrm{mn}$, is typically $\mathscr{T} \approx 0.3 \mathrm{~s}$ to $0.5 \mathrm{~s}$. This characteristic duration is of the order of 0.1- 0.3 Kolmogorov time scale $\tau_{K}$ and within the order of magnitude of the times observed on the PIV sequences like those presented in Figure 9. As $\mathscr{T}$ decreases the number of favorable events increases up to 200 in the limit $\mathscr{T} \rightarrow 0$ (1 PIV image). This reinforces the idea that a finite exposition duration $\mathscr{T}$ to the criteria is a good assumption. Getting 30 favorable events with $\mathscr{T}=0$ would require to change the two previous criteria into

$$
u_{d}=2.5 \times w_{s} ; \quad w_{d}=2.5 \times \frac{w_{s}}{\alpha}=2.5 \times \frac{w_{s}}{2}
$$

The Stokes response time of the particles while in suspension $\left(\tau_{p}=\frac{(\gamma-1) d^{2}}{18 v}=2 \times 10^{-5} \mathrm{~s}\right)$ is much smaller than the smallest flow times scales by the particle layer $\left(\tau_{K} \approx 1-3\right.$ $\mathrm{s}$ for $f=5 \mathrm{~Hz})$. This implies that as soon as they are lifted up, the particles behave like tracers, their velocity adjusting to the flow velocity. The vertical velocity component of the particles when ejected from the particle layer has been monitored and for $f=5 \mathrm{~Hz}$ ranges below $3 \mathrm{~mm} / \mathrm{s}$. This value and the scarcity of the ejections tend to confirm that the particles are not ejected by intense flow events (high velocity or shear) but that their lift up is more likely linked to long duration flow events.

The number of favorable velocity events at $f=4 \mathrm{~Hz}$ $\left(n_{f e_{-} 4 \mathrm{~Hz}}\right)$ and $f=6 \mathrm{~Hz}\left(n_{f e_{-} 6 \mathrm{~Hz}}\right)$ have also been counted. In each case the number of favorable events have been determined as previously: by counting the number of events that fulfilled criteria (18) and (20) with $\mathscr{T}$ within [0.3-0.5s] (impulse approach) or the modified criteria (24) with $\mathscr{T}=0 s$ (instantaneous approach). The concentrations $C_{6 \mathrm{~Hz}}$ and $\mathrm{C}_{4 \mathrm{~Hz}}$ associated with the numbers of favorable events determined with the "impulse approach": $n_{f e_{-} 4 \mathrm{~Hz}}$ and $n_{f e_{-} 6 \mathrm{~Hz}}$ respectively, have been determined from equation (23). They are plotted together with $\mathrm{C}_{5 \mathrm{~Hz}}$ in figure 7 (black squares). Concentrations thus estimated agree pretty well with the concentrations directly measured for this experiment. It gives a trend line (dotted line) that also satisfactorily reproduces the way concentration increases with the TKE-settling fluxes ratio. This result suggests that the main physics of the erosion process without mean flow is reasonably well captured by the "impulse approach". 


\section{CONCLUSION}

This study has been performed with the objective to describe the erosion of a particle layer when erosion is caused by a turbulence without mean flow and to identify the prevailing effects. For that, we have worked with a turbulence that is produced by a stirring grid located in a high position above the layer. The parameters that have been varied are essentially the size and the nature of particles (sawdust, PMMA), the turbulent energy produced to set particles in suspension and the buoyancy of the particles by changing their density ratio with the fluid. The flow between the grid and the particle layer has been characterized by velocity measurements (ADV, PIV). It is shown that the evolution of TKE measured from the grid follows the modified version of the $k-\varepsilon$ model of Matsunaga et al. ${ }^{1}$ that we propose to correct the inconsistency of this model over the range of viscosity and bulk Re number investigated here. The concentration in particles in the tank for various flow conditions has been measured through multiple points sampling and OBS. In steady regime, the concentration is homogeneous in the whole tank. It is found that all the measured concentrations exhibit an overall behavior when plotted as a function of the ratio between the flux of TKE towards the particle bed and the particle settling flux. The mechanisms involved in mobilizing particles from the layer have studied. Referring to "impulse approach" studies, we have postulated that to be eroded the particle should be first set in horizontal motion, before being lifted up and that the forces causing these motions should apply for a specific duration to be determined. The particle being embedded in the viscous sublayer a laminar criterion has been kept for horizontal motion (viscous drag versus friction), while an equilibrium between lift, drag and buoyancy has been considered for vertical motion. The obtained lift up criterion depends on the size of the particles relative to the viscous sublayer thickness and accounts for the shape of the deformed structures as they approach the particle layer. The characteristic duration of application of the forces required for lift up has been determined from our detailed $5 \mathrm{~Hz}$ experiments and used for the other frequencies. The duration found yields a number of eroded particles, hence concentrations that are in agreement with the measured concentrations in the bulk. It also gives a trend line that satisfactorily reproduces the way concentration increases with turbulent-settling fluxes ratio.

${ }^{1}$ N. Matsunaga, Y. Sugihara, T. Komatsu, and A. Masuda, "Quantitative properties of oscillating-grid turbulence in a homogeneous fluid," Fluid Dyn. Res. 25, 147-165 (1999).

${ }^{2}$ A. Shields, "Application of similarity principles and turbulence research to bed-load movement," Miteilungen der Preussischen Versuchsanstalt fur Wasserbau und Schiffbau (1936).

${ }^{3}$ P. Y. Julien, Erosion and sedimentation (Cambridge University Press, 2010).

${ }^{4}$ D. Kaftori, G. Hetsroni, and S. Banerjee, "Particle behavior in the turbulent boundary layer. i. motion, deposition, and entrainment," Phys. Fluids $\mathbf{7}$ 1095-1106 (1995).

${ }^{5}$ Y. Niñto and M. H. Garcia, "Experiments on particle-turbulence interactions in the near-wall region of an open channel flow: implications for sediment transport," J. Fluid Mech. 326, 285-319 (1996).
${ }^{6}$ I. Vinkovic, D. Doppler, J. Lelouvetel, and M. Buffat, "Direct numerical simulation of particle interaction with ejections in turbulent channel flows," Int. J. Mult. Flow 37, 187-197 (2011).

${ }^{7}$ E. J. Hopfinger and J.-A. Toly, "Spatially decaying turbulence and its relation to mixing across density interfaces," J. Fluid Mech. 78, 155-175 (1976).

${ }^{8}$ J. Yan, N.-S. Cheng, H.-W. Tang, and S. K. Tan, "Oscillating-grid turbulence and its applications: a review/turbulence de grille oscillante et ses applications: une revue," J. Hydr. Res. 45, 26-32 (2007).

${ }^{9}$ I. P. D. D. Silva and H. J. S.Fernando, "Oscillating grids as a source of nearly isotropic turbulence,” Phys. Fluids 6, 2455-2464 (1994).

${ }^{10} \mathrm{M}$. W. McCorquodale and R. J. Munro, "Experimental study of oscillatinggrid turbulence interacting with a solid boundary," J. Fluid Mech. 813, 768798 (2017)

${ }^{11}$ M. W. McCorquodale and R. Munro, "Analysis of intercomponent energy transfer in the interaction of oscillating-grid turbulence with an impermeable boundary," Phys. Fluids 30, 015105 (2018).

${ }^{12} \mathrm{M}$. W. McCorquodale and R. J. Munro, "A method for reducing mean flow in oscillating-grid turbulence,” Exp. Fluids 59, 182 (2018).

${ }^{13}$ H. J. S.Fernando and I. P. D. D. Silva, "Note on secondary flows in oscillating-grid, mixing-box experiments," Phys. Fluids A: Fluid Dyn. 5, 1849-1851 (1993).

${ }^{14}$ H. E. Huppert, J. S. Turner, and M. A. Hallworth, "Sedimentation and entrainment in dense layers of suspended particles stirred by an oscillating grid,” J. Fluid Mech. 289, 263-293 (1995).

${ }^{15} \mathrm{~N}$. Gratiot, H. Michallet, and M. Mory, "On the determination of the settling flux of cohesive sediments in a turbulent fluid," J. G. Res.: Oceans $\mathbf{1 1 0}$ (2005).

${ }^{16} \mathrm{H}$. Matinpour, S. Bennett, J. Atkinson, and M. Guala, "Modulation of time-mean and turbulent flow by suspended sediment," Phys. Rev. Fluids 4, 074605 (2019).

${ }^{17}$ R. E. Honey, R. Hershberger, R. J. Donnelly, and D. Bolster, "Oscillatinggrid experiments in water and superfluid helium,” Phys. Rev. E 89, 053016 (2014).

${ }^{18}$ L. Verso, M. van Reeuwijk, and A. Liberzon, "Steady state model and experiment for an oscillating grid turbulent two-layer stratified flow," Phys. Rev. Fluids 2, 104605 (2017).

${ }^{19}$ L. San, T. Long, and C. C. K. Liu, "Algal bioproductivity in turbulent water: An experimental," Water 9 (2017).

${ }^{20}$ T. Lacassagne, S. Simoëns, M. El Hajem, and J.-Y. Champagne, "Oscillating grid turbulence in shear-thinning polymer solutions," Phys. Fluids 31, 083102 (2019).

${ }^{21}$ T. Lacassagne, A. Lyon, S. Simoëns, M. El Hajem, and J.-Y. Champagne, "Flow around an oscillating grid in water and shear-thinning polymer solution at low reynolds number," Exp. Fluids 61 (2020).

${ }^{22}$ H. Traugott, T. Hayse, and A. Liberzon, "Resuspension of particles in an oscillating grid turbulent flow using PIV and 3D-PTV," in J. Phys.: Conference Series, Vol. 318(5) (IOP Publishing, 2011) p. 052021.

${ }^{23} \mathrm{H}$. Traugott and A. Liberzon, "Experimental study of forces on freely moving spherical particles during resuspension into turbulent flow," Int. J. Multiphase Flow 88, 167-178 (2017)

${ }^{24}$ C. H. Tsai and W. Lick, "A portable device for measuring sediment resuspension,” J. Great Lakes Res. 12, 314-321 (1986).

${ }^{25} \mathrm{~N}$. Gratiot, Etude expérimentale de la formation des couches de crème de vase turbulentes, Ph.D. thesis, Univ. J. Fourier, Grenoble (2000).

${ }^{26}$ N. Gratiot, M. Mory, and D. Auchère, "An acoustic doppler velocimeter (adv) for the characterisation of turbulence in concentrated fluid mud," Cont. Shelf Res. 20, 1551-1567 (2000).

${ }^{27}$ M. Rastello, Etude de la dynamique des avalanches de neige en aérosol, Ph.D. thesis, Université Joseph-Fourier-Grenoble I (2002).

${ }^{28} \mathrm{H}$. Michallet and M. Mory, "Modelling of sediment suspensions in oscillating grid turbulence," Fluid Dyn. Res. 35, 87 (2004).

${ }^{29}$ M. Rastello, H. Michallet, and J.-L. Marié, "Sediment erosion in zeromean-shear turbulence," in Costal Dyn. (2017).

${ }^{30}$ M. Ura, T. Komatsu, and N. Matsunaga, "Entrainment due to oscillatinggrid turbulence in two-layered fluid," Turbulence Measurements and Flow Modeling, 109-118 (1987).

${ }^{31}$ I. P. D. D. Silva and H. J. S. Fernando, "Some aspects of mixing in a stratified turbulent patch,” J.Fluid Mech. 240, 601-625 (1992). 
${ }^{32}$ S. M. Thompson and J. S. Turner, "Mixing across an interface due to turbulence generated by an oscillating grid," J. Fluid Mech. 67, 349-368 (1975).

${ }^{33}$ C. Goepfert, J.-L. Marié, D. Chareyron, and M. Lance, "Characterization of a system generating a homogeneous isotropic turbulence field by free synthetic jets," Exp. in Fluids 48, 809-822 (2010).

${ }^{34}$ S. Pope, Turbulent flows (IOP Publishing, 2001).

${ }^{35}$ A. O. Celik, P. Diplas, C. L. Dancey, and M. Valyrakis, "Impulse and particle dislodgement under turbulent flow conditions," Phys. Fluids 22 046601 (2010).

${ }^{36}$ R. J. Munro, N. Bethke, and S. B. Dalziel, "Sediment resuspension and erosion by vortex rings," Phys. Fluids 21, 046601 (2009).

${ }^{37}$ N. Bethke and S. B. Dalziel, "Resuspension onset and crater erosion by a vortex ring interacting with a particle layer," Phys. Fluids 24, 063301
(2012).

${ }^{38}$ T. Loiseleux, P. Gondret, M. Rabaud, and D. Doppler, "Onset of erosion and avalanche for an inclined granular bed sheared by a continuous laminar flow," Phys. Fluids 17, 103304 (2005).

${ }^{39}$ M. Ouriemi, P. Aussillous, M. Medale, Y. Peysson, and E. Guazzelli, "Determination of the critical shields number for particle erosion in laminar flow," Phys. Fluids 19, 061706 (2007).

${ }^{40}$ E. Guyon, J.-P. Hulin, L. Petit, and C. D. Mitescu, Physical hydrodynamics (Oxford University Press, 2015). 